\title{
A 15 AÑOS DE LA REVOLUCIÓN PINGÜINA ¿EN QUÉ ESTÁN LAS REFORMAS ESTRUCTURALES EN EDUCACIÓN?
}

\section{YeARS AFter the PENGUIN REVOLUTION: WHERE ARE THE STRUCTURAL REFORMS IN EDUCATION?}

Fecha recepción: 6 de octubre de 2021 / fecha aceptación: 10 de noviembre de 2021

\section{Juan Antonio Carrasco-Bahamonde ${ }^{1}$}

Cómo citar este artículo:

Carrasco-Bahamonde J. A. (2021). A 15 Años de la Revolución Pingüina ¿En qué están las reformas estructurales en educación? Revista Pensamiento y Acción Interdisciplinaria, 7(2), 27-45. https://doi.org/10.29035/pai.7.2.27

\section{Resumen}

El presente trabajo propone un análisis del ciclo de política educativa y cambio institucional en la educación chilena. Las movilizaciones de los estudiantes secundarios lograron conectar sus demandas con el cuestionamiento público del modelo educativo y la necesidad política de una reforma estructural. A partir de la revisión de literatura se propone una reconstrucción de la secuencia a través de la cual la problematización del mercado educativo es abordada en los planes de reforma. No obstante, el sentido ex post que asumen aquellas agendas de reforma, mantención y cambio, no son evidentes, suponen una sintaxis compleja donde los problemas de la política educativa se configuran como un campo en disputa. El artículo concluye que no es posible comprender el campo de producción de la política educativa en Chile en las últimas décadas, sin considerar la intensificación de la protesta estudiantil, aunque quedan preguntas abiertas sobre las perspectivas para futuras reformas regulativas orientadas a la equidad.

Palabras clave: Chile, movilizaciones estudiantiles, política de la educación, privatización, reforma educacional 


\begin{abstract}
This paper proposes an analysis of the cycle of educational policy and institutional change in Chilean education. Student mobilizations were able to connect their demands with the public questioning of the educational model and the political need for structural reform. Based on the literature review, a reconstruction of the sequence through which the problematization of the educational market is addressed in the reform plans is proposed. However, the ex-post sense that those reform agendas, maintenance, and change, are not evident, they assume a complex syntax where the problems of educational policy are configured as a field in dispute. The article concludes that it is not possible to understand the field of production of educational policy in Chile in recent decades without considering the intensification of student protest, although questions remain open about the prospects for future equity-oriented regulatory reforms.
\end{abstract}

Keywords: Chile, educational reform, politics of education, privatization, student mobilizations

\title{
Introducción
}

Durante los últimos años, Chile inició una discusión sustantiva sobre las bases de su institucionalidad educativa. Un ciclo creciente de movilizaciones sociales y protestas estudiantiles colocó en entredicho el carácter excesivamente mercantil y los efectos segregadores del sistema educativo, asentando la necesidad política de una reforma estructural y transformando el marco de expectativas en el que se ha desenvuelto el debate educacional (Aguilera y Álvarez, 2015; Bellei et al., 2011; Bellei, 2015; Carrasco-Bahamonde J. A. y Carrasco-Bahamonde D. A., 2018; Corvalán y García-Huidobro, 2015; Donoso, 2013; Donoso et al., 2014; Falabella, 2015). Estas circunstancias propician un nuevo campo de oportunidades invitando a repensar el rol de la educación pública como un horizonte democratizador para la sociedad chilena. Asimismo, comprender los mecanismos que están en la base de las desigualdades socioeducativas supone explorar las formas de regulación de los sistemas educacionales e indagar en el ciclo de la política educativa.

Por estas razones, preguntarse por la política de la educación, es decir, cómo se vinculan los sistemas educativos con los problemas de legitimación y crisis, está en el centro del interés público y académico. Este artículo brinda un abordaje analítico de especial relevancia para el estudio del proceso de reforma educativa, que permite situar en tensión el supuesto de la política estatal como expresión de un consenso racional, vincular las dinámicas de crisis a las características institucionales del sistema educativo y visibilizar la trama de intereses que confluyen en la elaboración de la política. De esta manera, se problematiza la periodización del ciclo reciente, ofreciendo una síntesis de los retos que actualmente enfrenta la política educativa al relevar la dimensión más política de la reforma educativa.

Para responder a este objetivo, se realizó una revisión sistemática de dos tipos de literatura. Se analizaron las iniciativas legales y otros registros oficiales, incluyendo la prensa de la época y, por otro lado, los estudios empíricos que 
encuadran los temas centrales de la reforma y ofrecen una primera estimación de su puesta en marcha (Bellei, 2015, 2016; Bellei y Muñoz, 2021; Muñoz y Weinstein, 2019; Zancajo, 2019). De este modo, el artículo busca brindar una aproximación más atenta a los procesos de generación de política precisamente en un contexto de eclosión de una nueva institucionalidad.

El texto se organiza en tres apartados. En el primero, se brinda una conceptualización para el abordaje de la política educativa a partir de las contribuciones de Dale (1989), Habermas (1986) y Offe (1984) y de la sociología de la política educativa. En esta perspectiva, la política institucional se encuentra atenazada tanto por la necesidad de asegurar las condiciones para la expansión del ciclo económico y los problemas de legitimidad, requerimientos que definen los bordes en que se mueve la política. En la segunda sección, se analizan cuáles han sido las proyecciones de las demandas de los movimientos estudiantiles, cómo estas son instituidas e insertas en una agenda y cómo finalmente los actores sociales desarrollan una capacidad diferenciada para producir -más o menos gradualmente- el cambio institucional. Finalmente, en un tercer apartado, se presenta un conjunto de conclusiones a modo de síntesis y retos para la política.

En suma, se sostiene que la forma cómo se modifica la naturaleza de las expectativas en torno a los sistemas educativos es también un proceso político y cultural, es decir, cómo los fines genéricos del sistema educativo se traducen en expectativas movilizadoras y socialmente legítimas supone la formación de una agenda específica, a partir de las reivindicaciones que impulsan los movimientos sociales, pero también sobre la base de un repertorio de rutinas institucionales, capacidades y disposiciones culturales que configuran un campo en disputa.

\section{Marco referencial: sociología de la política educativa}

La aproximación al estudio de la política educativa que suscribe este trabajo presenta un énfasis menos centrado en cuestiones administrativas o aspectos de implementación educacional. No busca solo servir para brindar soluciones a problemas ya dados, sino también visibilizar nuevas tensiones o dimensiones de las dificultades que pueden estar por fuera de la órbita de los tomadores de decisiones, en el diseño de políticas educacionales (Dale, 1994). Mientras que los estudios macropolíticos de la educación tienden a ocuparse más directamente de cómo los sistemas educativos responden a los imperativos del ciclo económico y las necesidades del sistema político, las investigaciones micro de la política se mueven en torno de cómo esta contribuye a reestructurar la práctica educativa y "entra", de alguna manera, a la sala de clases (Ball, 1994; Taylor et al., 1997).

Las transformaciones de los modos de regulación de la provisión escolar, cómo deben organizarse las políticas y cuál es su capacidad efectiva de permear la práctica educativa, han estado en el centro del debate educacional durante las últimas décadas. Los enfoques críticos se han centrado en los límites de las 
reformas y advierten que la capacidad que tienen los sistemas educativos de revertir desigualdades de origen es reducida dadas las condiciones estructurales en que se insertan. Si bien estos abordajes críticos poseen un diagnóstico común sobre los sistemas educativos, existe discrepancia respecto del mecanismo a través del cual se vehiculizan los efectos del sistema escolar en la estratificación social. Mientras que la educación puede ser comprendida como un valioso capital cultural en un mercado de bienes simbólicos, también lo ha sido como un transmisor de roles de subordinación y conformismo social (Collins, 1989).

La perspectiva que elabora Dale $(1989,1994)$ constituye una alternativa a los modelos determinísticos del cambio educativo y sostiene que la política de la educación se conecta más directamente con los problemas centrales del estado. Estos problemas consisten en asegurar las condiciones para la reproducción del ciclo económico, contener sus amenazas potenciales y cuestiones de legitimidad, es decir, con los contextos de poder más amplios en que se desenvuelve la política educativa (Dale, 1989, 1994). Este enfoque ofrece una manera de abordar el estudio de las reformas educacionales a partir del análisis del modo cómo se vinculan las formas del poder estatal a los sistemas de educación como un conjunto estructurado de instituciones, dotado de sus propias lógicas, con sus tensiones: "Fundamentally, the politics of education is the process and structure through which macro-societal expectations of education as institution are identified and interpreted and constituted as an agenda for the educational system" (Dale, 1994, p. 36).

Las respuestas a estos problemas supone traducirlos a través de repertorios institucionales y mecanismos que hacen de la política educativa un ensamblaje complejo y no necesariamente coherente (Dale, 1989; Offe, 1984). Como veremos en lo que sigue, el proceso de generación de política educativa que llevan a cabo las instituciones públicas del Estado debe enfrentar un doble reto: responder a aquellas agendas del sistema educativo y, a partir de esto, definir la organización de sus recursos y sortear tensiones internas viabilizando hasta cierto punto su abordaje.

En esta perspectiva, la política institucional se sitúa en medio de dos dinámicas interdependientes de crisis: la tendencia de crisis económica y el problema del orden (Dale, 1989). Estas tendencias de crisis se traducen en una presión creciente a las instituciones y definen los márgenes de lo posible de la política educativa. Las discrepancias que suscitan estas expectativas son sistemáticas y plantean una disyunción en la estructura del aparato administrativo del Estado, de tal modo que estos requerimientos van estrechando el ángulo de mira del Estado y reduciendo su zona efectiva de intervención. A partir de este abordaje, no existiría un camino no problemático de integración de los retos de la intervención del Estado y las discordancias que producen estos problemas, expansión del ciclo económico y legitimidad del orden político, que al interior del campo institucional son sistemáticas (Dale, 1989; Habermas, 1986; Offe, 1984). 


\section{Figura 1}

Diagrama del Sistema Político y las Tendencias de Crisis

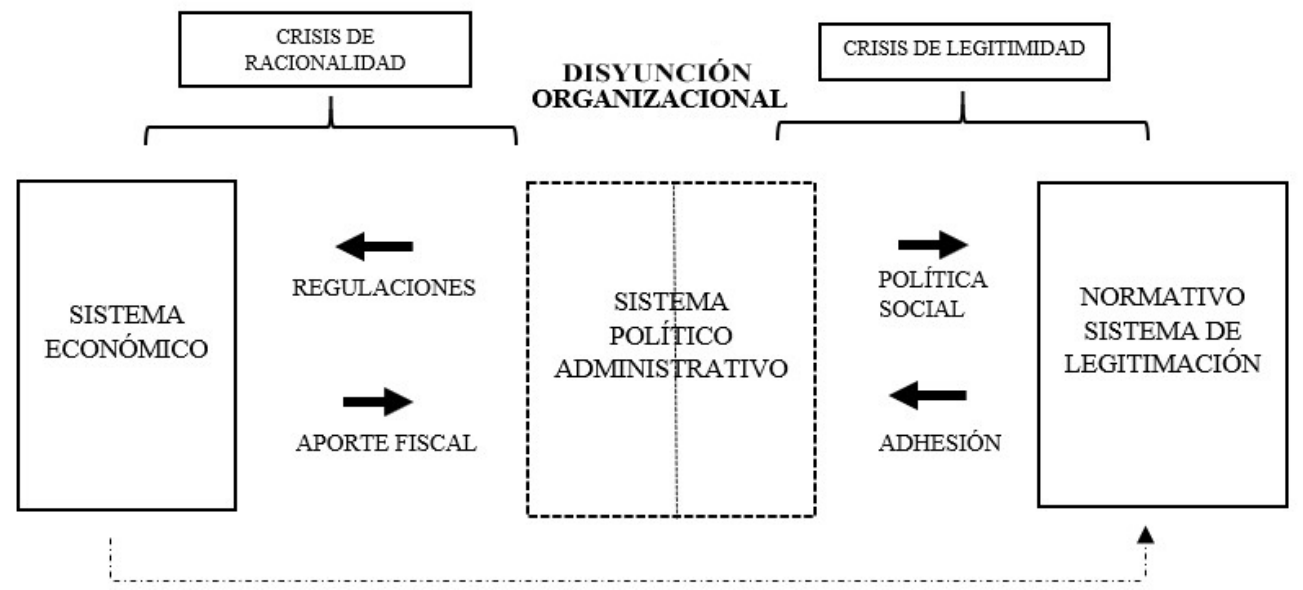

Fuente: Habermas, 1986; Offe, 1984

Las tendencias de crisis del subsistema político se pueden expresar como déficit de racionalidad (output) o de legitimidad (input), en relación con los subsistemas que interactúa. La crisis de legitimidad (el grado de adhesión de los ciudadanos expresado de manera difusa, que no depende de un juicio preciso) implica que los medios administrativos no logran mantener las estructuras normativas necesarias para asegurar la adhesión al orden institucional. En contextos de crisis, los medios administrativos y recursos regulatorios resultan insuficientes y se produce un proceso de autoobstrucción acumulativa cada vez mayor: "La crisis de racionalidad es una crisis sistémica desplazada; al igual que la crisis económica, expresa como contradicción de imperativos de autogobierno, la contradicción inherente a una producción socializada que se realiza en favor de intereses no generalizables" (Habermas, 1986, p. 65).

Antes que la dinámica desestabilizadora de los conflictos sociopolíticos ponga en riesgo los procesos de integración social, la intervención política se intensifica en aquellas áreas donde es más probable la irrupción del conflicto estructural, intenta aislar aquellos núcleos y contener su escalamiento. Esto define el carácter selectivo de la intervención de las instituciones estatales en la generación de política social (Habermas, 1986; Offe, 1984).

Dicho de otro modo: el costo en el manejo político de la crisis es una carga interventora cada vez mayor, de tal modo que se tienden a generar nuevos problemas, incluyendo la falta de adhesión hacia el sistema político. Además, de la contradicción primaria entre los requerimientos sistémicos que percibe el sistema político-administrativo se produce una crisis agregada, relacionada con su ordenamiento interno que da soporte a la política, el carácter insuficiente de los instrumentos y recursos organizacionales del Estado para enfrentar una 
complejidad y dificultad creciente. Estas discrepancias en el terreno de la política institucional se traducen en un déficit de racionalidad en el uso de los medios administrativos, regulatorios y la autoridad ${ }^{2}$ (Offe, 1984).

En ocasiones, como advierte Ball (1994), una política o un aspecto de una misma iniciativa puede conectar más directamente con uno u otro de estos requerimientos, de tal modo que esta doble funcionalidad provoca controversia y un déficit de coherencia. Sin embargo, no es posible suponer la consistencia del entramado de políticas educativas ni sobreestimar su capacidad de prescribir aquello que los actores educativos deben realizar para responder a los planes de reforma. En suma, el campo de la política versa de opciones en disputa, cuyos efectos diferenciales son contestados por los actores (Ball, 1994). Estarían precisamente en los intersticios de las instituciones, en sus márgenes y, especialmente, en las zonas de incerteza, donde se desarrollan más activamente procesos de disputa de los sentidos, recursos y la dirección de las organizaciones (Collins, 1989).

Las crisis de legitimación suponen procesos de descomposición institucional y de los medios administrativos a través de los cuales se ejerce el control burocrático, la erosión de las estructuras normativas y, en el largo plazo, un déficit de integración social. En este sentido, es relevante explorar cuánto de la política educativa responde más directamente a los requerimientos del ciclo productivo o, por otro lado, a los problemas de legitimación como son la expansión de derechos sociales y la profundización de la vida democrática. Asimismo, es preciso estimar el nivel de discrepancia entre estos propósitos en un contexto y trayectoria de políticas específicos. Al respecto, la política educativa articula de manera provisoria arreglos institucionales y un abordaje de la crisis, sujeto a rendimientos variables y múltiples escenarios de cambio (Dale, 1989).

Justamente, es en las coyunturas críticas de redefinición de las formas de control sobre el sistema educativo cuando se abren nuevas posibilidades para la política educativa. Por tanto, debemos preguntarnos sobre las implicancias de la redefinición de la provisión educativa y cómo se organiza el proceso de generación de las políticas. Así, el estudio crítico no puede eludir cuestiones esenciales como cuál es la trama de intereses a los que sirve la política, los grupos de influencia que se movilizan a través de medios no formales para incidir en su curso, cuán explícitos son estos intereses y cuál es la definición normativa que opera en torno de los actores.

\footnotetext{
2 Para Offe (1984) los principales recursos regulatorios son el gasto fiscal, la racionalidad administrativa y la lealtad de las masas. Las decisiones presupuestarias en torno a ingresos y gastos fiscales se deben analizar a partir de un doble criterio: al mismo tiempo, permiten generar condiciones para mantener el proceso de acumulación y, por otro, obstaculizarlo a partir del uso no productivo de recursos en el sentido capitalista (p. 57). Los ingresos que demanda la expansión del gasto fiscal suponen interrumpir parcialmente el proceso de acumulación a través de la conversión en impuestos, tarifas y préstamos fiscales de una porción del producto interno bruto destinado a la inversión. El problema que le plantea al Estado la entrega de subsidios y el pago de transferencias a través de las políticas es que pasan a ser parte del gasto permanente y su rendimiento estabilizador es decreciente.
} 
Este abordaje conceptual permite situar en tensión el supuesto de la política educativa como expresión de un consenso racional, donde distintos actores confluyen sobre unos propósitos por fuera de las disputas y la trama de intereses en juego. Más bien, busca comprender aquel entramado como un ensamblaje complejo, no necesariamente coherente y sujeto a múltiples requerimientos. Estará en los contextos de crisis, donde se redefinan los términos de la relación entre el poder institucional, el sistema educativo y las agendas de cambio o estabilización que promueven los actores sociales y políticos.

\section{¿Y las reformas estructurales? Análisis del ciclo de políticas en la educación chilena}

El periodo autoritario se caracterizó por un cambio en la naturaleza del estado, su relación con el sistema educativo y sus esfuerzos por desmantelar el Estado de Compromiso que permaneció en la base de la promoción popular. En una primera etapa, el énfasis fue asegurar el orden y el control geopolítico, dando paso a una dimensión proyectual del régimen, que apostó definitivamente por una integración de políticas conservadoras y neoliberales, centradas en el dinamismo del sector primario exportador de la economía.

En este contexto, Chile fue una de las reformas más ambiciosas, la que se fundamentó en la elección escolar, tanto por la radicalidad de su implementación, su escala universal y extensión en el tiempo. En 1981 se impulsó una transformación educativa sin precedentes, a través de una reforma que disminuyó las barreras para la formación de oferentes privados, redujo el tamaño del sector público y ajustó de manera severa el gasto fiscal. La concreción de esta modernización -como se denominó en el lenguaje de la época- se instruyó con el DFL 1-3063 (1980) que, además, puso en marcha la descentralización de la gestión administrativa de las escuelas públicas, traspasándola a los municipios, como también la implementación de un sistema de financiamiento a la demanda, que en la práctica homologa la subvención general a escuelas públicas y privadas que perciben el beneficio estatal. La formación de este mercado masivo de la educación buscó asegurar el acceso efectivo y consagró constitucionalmente la libertad de enseñanza como principio rector (Orellana, 2018).

Al comenzar la década de los 80's, la dictadura enfrentaba un grave escenario de crisis económica e importantes retos de legitimidad y estabilización política del régimen. Si bien la crisis económica posee factores internacionales, sus efectos en el desempleo masivo y el incremento de la pobreza se profundizan a partir del ajuste del gasto social y las políticas monetaristas que el propio régimen impulsaba de manera más ortodoxa en una primera etapa. En este contexto, se postergan los programas de modernización social y la reforma educativa que habían sido anunciados en el año 1979 a través de distintos medios. 
A inicios de 1980 se decretó la iniciativa legislativa DL. 3166 (1980), que faculta al Ministerio de Educación para el traspaso de establecimientos educacionales a entidades privadas. A partir de esto, la autoridad promovió un rol activo de los gremios en la provisión educativa y facilitó el traspaso de 70 establecimientos educacionales, preferentemente liceos de formación técnica, a 21 asociaciones empresariales que se constituyen como corporaciones de administración delegada, contando con un financiamiento público que no posee un cálculo como la subvención general, sino más bien es asegurado y con escaso control público (Almonacid, 2004).

El gobierno buscó revestir de institucionalidad las medidas que se habían tomado de facto e iniciar un conjunto de modernizaciones en materia social, como serían las reformas de los sistemas de educación, salud y pensiones. Para esto, establece un nuevo marco legal tras un plebiscito realizado en un contexto de serias restricciones civiles. La nueva constitución de 1980 consagró la libertad de enseñanza y de elección como principios rectores de la educación. Este nuevo orden constitucional va a concretar el derecho a la educación como libertad de enseñanza, es decir, el derecho de los proveedores educativos a establecerse y otorgará a la familia la responsabilidad de orientar la educación por sobre el Estado, entendiendo esta prerrogativa como la libertad de las familias para elegir entre distintas instituciones.

En términos generales, podemos establecer una periodización reconociendo este momento de instalación de las bases institucionales en contexto autoritario entre 1980-1987, que reemplaza el modelo de provisión estatal conocido como "Estado Docente" (Bellei y Muñoz, 2021). Tras esto, durante la década de los 90's, se desarrolla un ciclo de políticas educacionales que restituye el rol compensatorio del Estado a partir de la expansión del gasto público, la modernización del currículum educativo y la transformación de los contextos pedagógicos donde interactúan profesores y estudiantes. En este marco, y a partir del año 2006, escala el proceso de movilizaciones estudiantiles y la crítica del andamiaje institucional de la educación chilena, adquiriendo notoriedad en el debate público. En 2014 se inicia un ciclo de reforma, orientado a la revisión del diseño institucional que busca activamente revertir los efectos del mercado en la estratificación socioeconómica del sistema educativo. 
El siguiente esquema presenta una síntesis de la trayectoria de política educativa y los énfasis de los distintos periodos:

\section{Figura 2}

Síntesis de Modos de Regulación del Sistema Educativo

\begin{tabular}{|c|c|c|c|c|}
\hline ESTADO DOCENTE & $\begin{array}{c}\text { MERCADO } \\
\text { DESREGULADO }\end{array}$ & $\begin{array}{l}\text { “DOS LÓGICAS } \\
\text { PARALELAS" }\end{array}$ & ESTADO EVALUATIVO & REFORMA PROEQUIDAD \\
\hline Hasta 1980 & 1980-1990 & 1990-2006 & 2006-2014 & 2014 al presente \\
\hline $\begin{array}{l}\text { El Estado chileno es el } \\
\text { principal proveedor de } \\
\text { educación: el sector } \\
\text { privado "coopera" con el } \\
\text { Estado en los fines } \\
\text { educativos, lo que } \\
\text { garantiza la libertad de } \\
\text { enseñanza. }\end{array}$ & $\begin{array}{l}\text { La educación pública es } \\
\text { traspasada a la } \\
\text { administración municipal y } \\
\text { compite con los nuevos } \\
\text { administradores privados: } \\
\text { se promueve el } \\
\text { financiamiento a la } \\
\text { demanda, la elección de } \\
\text { escuela y reformas } \\
\text { privatizadoras. }\end{array}$ & $\begin{array}{l}\text { Se mantienen las bases } \\
\text { institucionales de la } \\
\text { educación en torno a la } \\
\text { competencia y elección } \\
\text { escolar. } \\
\text { Un nuevo rol } \\
\text { compensatorio del estado a } \\
\text { través de programas a } \\
\text { escuelas desfavorecidas. } \\
\text { Inclusión de un copago } \\
\text { familiar como mecanismo } \\
\text { de financiación educativa al } \\
\text { sector privado } \\
\text { subvencionado. }\end{array}$ & $\begin{array}{l}\text { Tras la Revolución } \\
\text { Pingüina se crea el } \\
\text { Consejo Asesor } \\
\text { Presidencial y se propone } \\
\text { un nuevo marco para la } \\
\text { educación. } \\
\text { La Ley General de } \\
\text { Educación sustituye a la } \\
\text { LOCE. } \\
\text { Ampliación de las pruebas } \\
\text { e incentivos basados en el } \\
\text { rendimiento. }\end{array}$ & $\begin{array}{l}\text { Ley de Inclusión regula y } \\
\text { prohíbe el lucro, el copago y } \\
\text { la selección como prácticas } \\
\text { discriminatorias. } \\
\text { Política de } \\
\text { desmunicipalización y } \\
\text { fortalecimiento de la } \\
\text { educación pública por parte } \\
\text { de los nuevos servicios } \\
\text { locales de educación. } \\
\text { Proyecto de nueva carrera } \\
\text { docente. }\end{array}$ \\
\hline & & $\Gamma------7$ & & $\Gamma------$ \\
\hline MODELO BUROCRÁTICO & REFORMAS DE MERCADO & {$\left[\begin{array}{c}\text { MARCO } \\
\text { REGULATORIO }\end{array}\right.$} & RENDICIÓN DE CUENTAS & $\begin{array}{l}\Gamma-\text { ESTADO PROVEEDOR } \\
\text { - - - - - }\end{array}$ \\
\hline
\end{tabular}

Fuente: Bellei y Vanni, 2015; Bellei y Muñoz, 2021

A partir de estas reformas, el sistema educativo chileno poseerá cuatro tipos principales de provisión: escuelas públicas administradas por los municipios, escuelas particulares subvencionadas con fondos públicos, escuelas particulares financiadas exclusivamente por el pago de las familias y un conjunto de instituciones de formación técnica que fueron traspasadas a los gremios empresariales como corporaciones de administración delegada. 
A 15 Años de la Revolución Pingüina ¿En qué están las reformas estructurales en educación? por Juan Antonio Carrasco-Bahamonde

\section{Figura 3}

Mecanismo de Financiamiento y Provisión de la Educación a partir de 1981

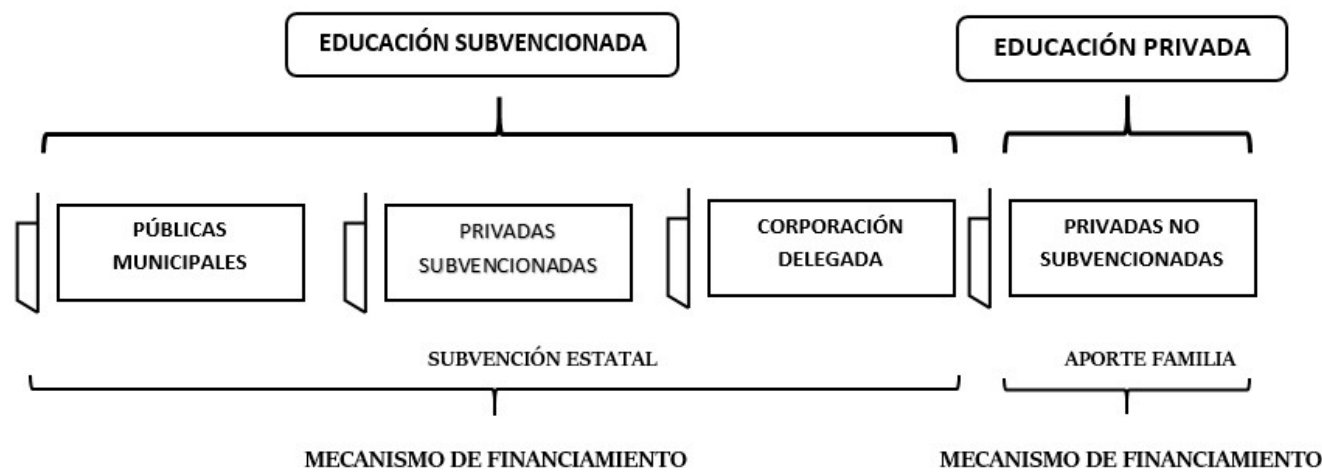

Fuente: Elaboración propia

El imaginario político del periodo que se inicia con la apertura democrática no problematiza el marco jurídico institucional en que se desenvuelve la actividad educativa. Los responsables de las políticas del periodo llaman a "desdramatizar" la distinción entre escuelas públicas y privadas, adoptando simplemente la arquitectura heredada. En un contexto institucional inestable y negociando con sus adversarios aún en el parlamento, para la política de este periodo la escuela sería justamente un núcleo privilegiado de las transformaciones que la educación chilena requiere en un proceso de democratización e inserción en el concierto internacional. En este marco, cobra relevancia un tipo de discurso más técnico-educacionista.

La estrategia de estos gobiernos se orientará preferentemente a la implementación de programas focalizados en un horizonte de cambio cultural desde las escuelas, sin modificar en lo sustantivo las bases institucionales del sistema educativo (Bellei y Vanni, 2015). Durante el primer bienio del gobierno democrático (1990-1991), se enfatizó en las condiciones laborales del profesorado y cómo revertir la situación de la educación pública municipal a partir de programas focalizados hacia la ruralidad y el 10\% las escuelas básicas que presentaban mayor deterioro en sus resultados (P900). Para Corvalán y García-Huidobro (2015) este nuevo ciclo de políticas educativas rompe con el "compromiso político ideológico" de la dictadura, poniendo en el centro los desafíos de la educación pública y la equidad social.

El año 1993, tras una negociación parlamentaria que incluyó una reforma tributaria, se introducen a través de la Ley de Impuesto a la Renta incentivos para un aporte de las familias como modalidad complementaria de financiamiento de las escuelas privadas subvencionadas y los liceos públicos que imparten enseñanza media. Estos lograban cobrar un pago sin perder la subvención estatal o reduciendo solo parcialmente su monto. Esta política fue ampliamente 
adherida por la educación privada subvencionada. En el año 1998, un 42\% de las escuelas que representaban un $72 \%$ de la matrícula del sector incluía algún nivel de financiamiento compartido, iniciando un proceso de diferenciación de la oferta privada subvencionada de acuerdo con la capacidad de pago de las familias (Almonacid, 2004).

La política de financiamiento compartido no tuvo mayor respuesta en el mundo de las escuelas públicas, en la práctica la incorporación del copago de las familias operó como un acelerador del proceso de fragmentación social y económica del sistema educativo.

\section{Figura 4}

Mecanismo de Financiamiento y Provisión de la Educación a partir de 1994

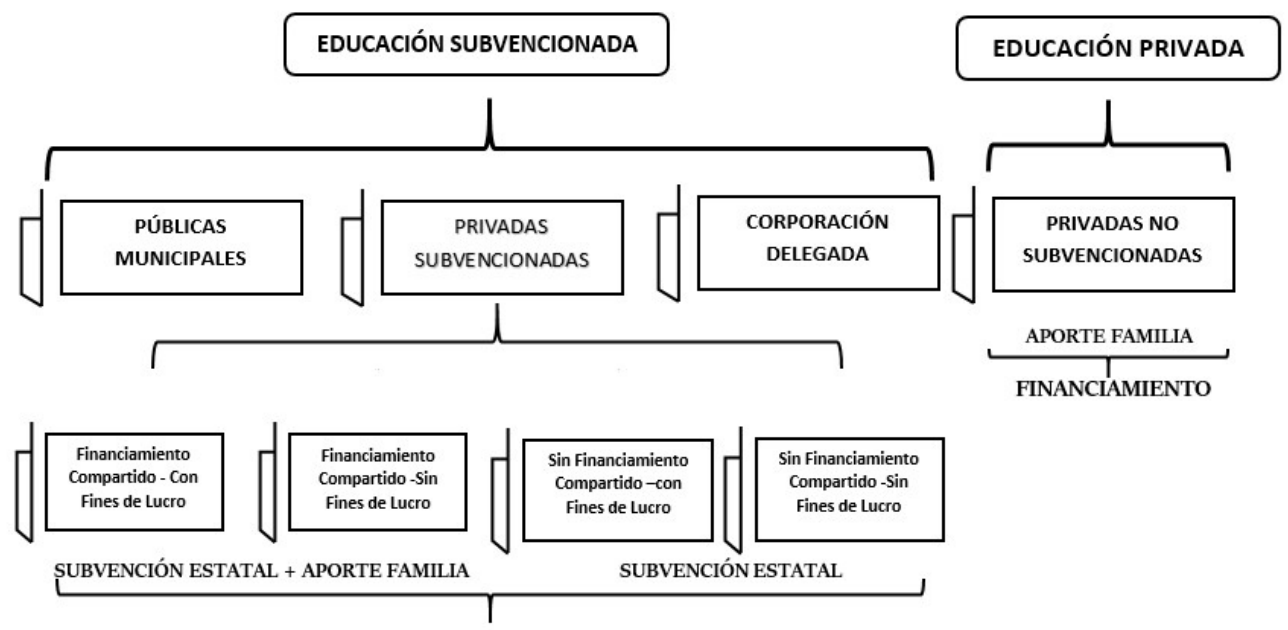

MECANISMO DE FINANCIAMIENTO

Fuente: Elaboración propia

El año 1994 marcó el curso para la política educacional del periodo. A inicios del segundo gobierno de la Concertación, el presidente Eduardo Frei convoca a un Diálogo Nacional sobre la Modernización de la Educación Chilena, para lo cual forma a través de un decreto la Comisión Nacional para la Modernización de la Educación que adopta la denominación de "Comisión Brunner", por quien fue su coordinador y Secretario General de Gobierno entre 1994-1998. El Comité Técnico Asesor de la Comisión estuvo compuesto por 17 representantes de diversos sectores, incluyendo entidades gremiales y centros de pensamiento ligados a todo el espectro político.

Después de una década de reforma educacional, la Organización para la Cooperación y el Desarrollo Económicos (OCDE, 2004) concluye que los bajos resultados y el estancamiento experimentado lleva a cuestionamiento el camino 
emprendido en torno a la expansión de los incentivos y mecanismos de mercado en el sistema educativo chileno. Asimismo, la revisión comprueba la amplia aceptación entre los responsables de la política educativa, quienes defendían la idea de que la competencia tipo mercado impacta positivamente en el rendimiento, eficiencia y efectividad de las escuelas, y medidas afines como incentivos salariales a los profesores en base al rendimiento en las pruebas estandarizadas y la subvención a las escuelas privadas como una "variante" de la educación pública en Chile:

\begin{abstract}
... A pesar del gran cambio político en 1990 de un gobierno militar a uno democrático, las reformas de mercado chilenas de los años ochenta han tenido un impacto sostenido en la forma de las intervenciones educacionales de los años noventa. La más importante de estas reformas, por supuesto, es el sistema de subvenciones donde el estado subsidia colegios privados esencialmente en el mismo nivel que a establecimientos municipales (p. 177) [...] La noción de que la privatización de los costos educacionales contribuyó a la "eficiencia" educacional también se incorporó profundamente a la cultura de la política educacional chilena. (p. 180)
\end{abstract}

En este clima, las críticas se centraron en los límites de la reforma educativa promovida por los gobiernos concertacionistas y la educación pasó a un segundo plano en las elecciones presidenciales del año 2006 (Bellei y Vanni, 2014). Sin embargo, lo que marcó la primera etapa del gobierno electo fue el ascenso del movimiento estudiantil secundario que, a partir de reivindicaciones específicas como la liberación de cobros al transporte público y la prueba de selección universitaria, impulsó un cuestionamiento en cómo la política educativa se había formulado hasta ese momento en el marco de un paradigma de excesiva mercantilización. Después de un intenso proceso de movilizaciones, el gobierno anunció la creación de un Consejo Asesor Presidencial para la Calidad de la Educación, para discutir posibles soluciones a los retos estructurales del sistema educativo, conteniendo en cierta medida la presión social que desataron las movilizaciones estudiantiles. La composición del Consejo, dio cuenta de un amplio espectro de voces que comprendió a estamentos de la comunidad educativa, académicos y actores institucionales, cuyo propósito fue generar propuestas para una reforma educativa que integrase, al menos parcialmente, la visión de los estudiantes.

Entre las principales conclusiones del Consejo, reconocen la necesidad de un nuevo marco regulatorio para avanzar hacia el fortalecimiento de la educación pública, que propiciase las bases de un sistema sustentado en derechos sociales garantizados como aspecto central de una nueva etapa en la reforma educacional. Es así como comenzó la tramitación de un conjunto de proyectos de ley que dan forma a una nueva reforma educativa (Donoso et al., 2014). Entre las iniciativas legislativas que impulsó el ejecutivo, a partir del año 2007, se contabilizan la Ley 
de Subvención Escolar Preferencial (2008), la Ley General de Educación (2009) y la Ley de Aseguramiento de la Calidad (2011), las que reconocen una condición desigual en la base de los aprendizajes de los estudiantes y reemplazan el marco institucional dado por la Ley Orgánica Constitucional de Educación (1990), decretada los últimos días de la dictadura. Este conjunto de propuestas se orientó hacia el avance de una "nueva arquitectura para la educación chilena" como parte de un acuerdo político transversal. A partir de este contexto, el marco de políticas tendió a crear y fortalecer instrumentos de rendición de cuentas, estándares y controles.

El año 2011 se intensificaron las movilizaciones estudiantiles por parte de los universitarios, retomando la crítica a la mercantilización de la educación. Es posible reconocer distintos factores que confluyeron en la problematización del mercado educativo. Un aspecto central es la acumulación de estudios sobre temas como la segregación socioeducativa, las prácticas de selección o los efectos del copago de las familias, que permitió contextualizar el debate a la luz de evidencia investigativa, lo que permitió dimensionar tanto las limitaciones de la institucionalidad como las consecuencias no deseadas del mercado en la educación y, posteriormente, facilitar el proceso de diseño de la reforma y sus tiempos de implementación (Muñoz y Weinstein, 2019; Zancajo, 2019). Este conocimiento acumulado sirvió a centros de pensamiento, organizaciones sociales y actores políticos para estructurar una crítica al modelo educativo basada en evidencias, para fortalecer un consenso sobre la necesidad de una reforma estructural que propicie una nueva institucionalidad.

Otra fuente de problematización del mercado educativo y sus consecuencias se dio en un ámbito más amplio de preocupaciones sobre la desigualdad social, en tanto se constituye en una barrera para el desarrollo social y económico más general en el país. El proceso de desarrollo y crecimiento económico basado en el dinamismo del sector primario exportador estaría mostrando claras señales de estancamiento y agotamiento estructural. El discurso experto de asesores influyentes a nivel político reconoció en el modo de regulación del sistema educativo una de las causas principales de la desigualdad social y la pérdida de dinamismo económico, favoreciendo su priorización en una agenda mayor de transformaciones. Finalmente, hay una motivación de tipo político-ideológico que radica en la necesidad de ampliar la base de apoyo electoral y la composición de la nueva coalición de gobierno incorporando a actores que, tradicionalmente, cuestionaron ese modelo de desarrollo. En este sentido, un aspecto relevante es que esta coalición amplia tuvo el control del poder legislativo entre 2014-2018, para la puesta en marcha de la reforma (Bellei, 2016; Muñoz y Weinstein, 2019; Zancajo, 2019).

Con estos antecedentes, el programa de gobierno de una nueva coalición "Nueva Mayoría" (2013) se planteó desarrollar reformas estructurales al sistema político, tributario y en educación como ejes centrales para su periodo. En este 
último ámbito, se propuso enfrentar los altos niveles de desigualdad y segregación social. La reforma estructural en el sistema educativo sería el "desafío primordial", según sus definiciones programáticas (Bachelet, 2013). A partir del año 2015, Chile adopta una nueva agenda de reforma educativa que se define especialmente por aquellos ámbitos que buscan regular, incluyendo cuatro dimensiones clave: (i) el fin del lucro en educación por parte de instituciones que perciben la subvención estatal; (ii) la reducción progresiva al copago de las familias a las escuelas particulares subvencionadas, a partir de su sustitución por un aporte estatal que permite sostener aquella provisión privada hasta cierto monto sin que las escuelas modifiquen su administración; (iii) evitar las distintas formas de selección de los estudiantes por parte de las escuelas durante todo el ciclo formativo y la implementación de un sistema de admisión escolar unificado; (iv) la puesta en marcha de una nueva institucionalidad para la educación pública que incluye el traspaso gradual de la gestión escolar desde los municipios a nuevos Servicios Locales de Educación que se caracterizan por una mayor autonomía, cobertura territorial y su carácter técnico educativo.

Aunque el gobierno inició estas reformas estructurales con un fuerte respaldo público, la reforma educativa despertó una fuerte oposición en diversos grupos de interés, especialmente los partidos de oposición, y cierto obstruccionismo incluso al interior de la coalición de gobierno, agrupaciones gremiales, sostenedores y en familias organizadas. Durante el proceso de preparación, en el que se discutió el alcance de la reforma, el debate se mediatizó y los medios de comunicación vinculados a los sectores más conservadores jugaron un rol activo en su tratamiento e impulsaron críticas a la iniciativa que fueron ganando terreno en la opinión pública. A partir de la primera propuesta de ley, enviada al Parlamento en mayo del año 2014, se producen una serie de cuestionamientos que provienen, especialmente, de asociaciones de escuelas privadas y familias organizadas.

Por otro lado, tanto el fin al lucro como la "desmunicipalización" de la educación pública han sido demandas centrales del movimiento estudiantil del 2011. En noviembre del año 2017 fue promulgada la Ley 21.040 (2017) que crea un nuevo Sistema de Educación Pública, que se traduce en la implementación de los nuevos Servicios Locales de Educación (SLE). El proceso de implementación de los SLE constituye un proceso gradual, iniciado el año 2018, a partir del cual se formarán setenta SLE en el marco del proyecto de Fortalecimiento de la Educación Pública, iniciativa que busca revertir el proceso de deterioro y crisis de la educación pública en Chile.

Rojas (2014) advierte que reimaginar la educación pública requiere más que un acto administrativo y de romper la trampa de la segmentación social del sistema educativo chileno. Para que las escuelas públicas puedan ser una alternativa para las clases media y alta debemos avanzar hacia una idea más clara del valor agregado que ofrecen y dejar atrás la medición estandarizada de los aprendizajes, como principal índice de la calidad esperada de las escuelas. Una agenda de 
reforma educativa que sitúa en el centro la inclusión social y sostener un modelo de rendición de cuentas basado en pruebas estandarizadas constituyen señales fuertemente contradictorias para las escuelas.

En sus primeros años de implementación, y a pesar de los propósitos de la reforma educativa, persisten las dinámicas de mercado desatadas en el sistema educativo, se activan nuevos grupos de interés contra el proceso de reforma y se abren distintos escenarios para las escuelas. De este modo, la experiencia chilena se torna particularmente relevante en un contexto internacional en el que diferentes organizaciones han señalado la necesidad de adoptar una regulación más efectiva, para evitar los efectos de los mercados educativos y la expansión de la oferta privada sobre la equidad, siendo uno de los primeros países en adoptar una reforma orientada a regular las políticas promercado en educación (Zancajo, 2019).

\section{Conclusiones}

La reforma ha sido el código en el que se organiza la política educacional durante las últimas décadas, el modo de traducción de las expectativas sociopolíticas en una diversidad de agendas de cambio y estabilización, transitando a través de distintos ciclos con rupturas y continuidades. En suma, procesos de profunda transformación de la sociedad chilena, marcados por una presión cada vez mayor hacia el sistema educativo. Como se elabora una agenda de política delimita el modo en que las expectativas contenidas serán eventualmente abordadas, de tal modo que aquellas presiones que experimentan los sistemas educativos no serían del todo evitables, incluyendo intereses que suelen ser contradictorios. Como contrapartida, el proceso de reforma define aquel alcance de transformaciones que ponen en marcha los actores institucionales, aquello que es esperable y, por vía negativa, aquello que queda fuera de alcance, cuáles son los medios administrativos, financieros y simbólicos disponibles, de tal manera que como se constituye la política no solo plantea los propósitos a seguir, sino también el modo en que estos pueden ser alcanzados.

La revisión anterior sugiere que las respuestas del sistema político institucional dieron paso a impulsos diferenciados de política educativa. Mientras que la reforma concertacionista impulsó una agenda intensa de programas orientados al contexto de las escuelas, con las movilizaciones del año 2006 se inicia un ciclo de reforma que buscó modificar el marco institucional más amplio. La trayectoria de regulación del sistema educativo se mueve desde un modelo fuertemente orientado al mercado, fortaleciendo mecanismos de control y estandarización evaluativa, en parte como una primera respuesta a las demandas que movilizaron los estudiantes. Este tipo de reforma regulatoria renueva la institucionalidad reemplazando la LOCE por la Ley General de Educación (Ley 20.370, 2009), crea una agencia de calidad y la superintendencia de educación, complejizando el modo de financiamiento de las escuelas a través de la SEP (Ley 20.248, 2008), 
que corrigió la subvención plana. Tras las movilizaciones de los estudiantes universitarios el 2011, se configuró una nueva agenda de reforma para regular de manera decidida el sector privado subvencionado y fortalecer la educación pública, situando la cuestión de la equidad en el centro de la agenda.

No obstante, persisten ciertos anclajes institucionales de difícil resolución. El esquema de financiamiento conservó su carácter competitivo, aunque incorporando criterios compensatorios. También se consolidó la formación de un sector privado subvencionado como eje de la provisión educativa que presiona hacia un sistema fuertemente mercantilizado y segmentado socialmente.

De este modo, en el nivel macro de la política educativa, se observa un déficit de coherencia a partir de incentivos y señales fuertemente contrapuestas. Un segundo tipo de desafíos radica en la sostenibilidad de la implementación del proceso de reforma en un contexto de cambio político. Después de dos décadas de continuidad, Chile inició un periodo de alternancia, formación de nuevas fuerzas políticas y cambios en la composición de las coaliciones gobernantes. Estos ciclos "cortos" han puesto en entredicho la sostenibilidad para la implementación de las reformas, al mismo tiempo que se expanden las expectativas sociales en torno a los sistemas educativos y la presión por el acceso a derechos sociales. En este sentido, cabe señalar que se activan grupos de interés que buscan obstaculizar el avance de las reformas estructurales en educación.

Un aspecto relevante ha sido la presencia de efectos no esperados y necesidad de corrección durante el camino. Un proceso de reforma de gran envergadura supone efectos no deseados como pueden ser la elitización o la segmentación "por arriba" de la provisión escolar. Finalmente, resulta plausible anticipar que dada la persistencia de los incentivos competitivos y pruebas estandarizadas como índice de la calidad, las escuelas que se beneficiaron de las dinámicas de mercado tenderán a codificar y generar nuevas estrategias de control sobre la composición de su matrícula y otros atributos sensibles a las familias.

En definitiva, no es posible comprender el campo de producción de la política educativa en Chile, sus posibilidades y límites, sin considerar la intensificación de los procesos de movilización y protestas estudiantiles que desplazan los marcos institucionales concitando el respaldo ciudadano. No obstante, los retos señalados representan aún preguntas abiertas sobre el avance de las reformas regulatorias y sus implicancias más amplias en la equidad. 


\section{Referencias bibliográficas}

Aguilera, O. y Álvarez, J. (2015). El ciclo de movilización en Chile 2005-2012: Fundamentos y proyecciones de una politización. Revista Austral de Ciencias Sociales, (29), 125-145. https://doi.org/10.4206/rev.austral.cienc.soc.2015. n29-07

Almonacid, C. (2004). Un cuasi-mercado educacional: La escuela privada subvencionada en Chile. Revista de Educación, (333), 165-196. http://hdl. handle.net/11162/67314

Bachelet, M. (2013). Programa de Gobierno Michelle Bachelet 2014-2018. http:// www.subdere.gov.cl/sites/default/files/noticias/archivos/programamb_1_0.pdf

Ball, S. (1994). Education Reform. A Critical and Post-structural Approach. Open University Press.

Bellei, C. (2015). El Gran Experimento. Mercado y Privatización de la Educación Chilena. LOM.

Bellei, C. (2016). Dificultades y resistencias de una reforma para des-mercantilizar la educación. RASE, 9(2), 232-247. https://ojs.uv.es/index.php/RASE/article/ view/8417/8010

Bellei, C. y Muñoz, G. (2021). Modes of Regulation, educational policies, and changes in education system: a long-term analysis of the Chilean case. Journal of Educational Change. https://doi.org/10.1007/s10833-021-09435-1

Bellei, C. y Vanni, X. (2015). Chile: The Evolution of Educational Policy 19802014. En S. Schwartzman (Ed.), Education in South America (pp.179-200). Bloomsburry Academic.

Bellei, C., Contreras, D. y Valenzuela, J. (2011). Ecos de la Revolución Pingüina. Avances, debates y silencios de la Reforma Educativa. Unicef, Universidad de Chile.

Carrasco-Bahamonde, J. A. y Carrasco-Bahamonde, D. A. (2018). Cartografía de la Reforma Educativa en Chile. Mercado, Competencia y Mejoramiento Escolar. Revista Brasileña de Educación, 23, e230080. https://doi.org/10.1590/S141324782018230080

Collins, R. (1989). La Sociedad Credencialista. Sociología histórica de la Educación y la Estratificación. Akal Universitaria.

Corvalán, J. y García-Huidobro, J. (2015). La educación de mercado en Chile y su propuesta de superación. Cuaderno de Educación, (66), 1-16. 
A 15 Años de la Revolución Pingüina ¿En qué están las reformas estructurales en educación? por Juan Antonio Carrasco-Bahamonde

https://repositorio.uahurtado.cl/bitstream/handle/11242/7357/articulo_4. pdf?sequence=1\&isAllowed $=y$

Dale, R. (1989). The State and Educational Policy. Open University Press.

Dale, R. (1994). Applied Education Politics or Political Sociology of Education? Contrasting Approaches to the Study of Recent Educational Reform in England and Wales. En D. Halpin y B. Troyna, (Eds), Researching Educational Policy. Ethical and Methodological Issues (pp. 31-41). Falmer Press.

Decreto Ley 3166 de 1980. Autoriza entrega de la administración de determinados establecimientos de educación técnico profesional a las instituciones o a las personas jurídicas que indica. 6 de Febrero de 1980.

Decreto 1-3063 de 1980 [con fuerza de ley]. Reglamenta aplicación inciso segundo del artículo $38^{\circ}$ del DL. №. 3.063, de 1979. 13 de Junio de 1980.

Donoso, S. (2013). Dynamics of Change in Chile: Explaining the Emergence of the 2006 Pingüino Movement. Jornal of Latin American Studies, 45(1), 1-29. https://doi.org/10.1017/S0022216X12001228

Donoso, S., Frites, C. y Castro, M. (2014). Los proyectos de ley de fortalecimiento de la educación pública de los años 2008 y 2011: propuestas y silencios. Revista Pensamiento Educativo, 51(2), 1-18. https://doi.org/10.7764/ PEL.51.2.2014.1

Falabella, A. (2015). El Mercado Escolar en Chile y el Surgimiento de la Nueva Gestión Pública: El Tejido de la Política entre la Dictadura Neoliberal y los Gobiernos de la Centroizquierda (1979 a 2009). Educação \& Sociedade, 36(132), 699-722. https://dx.doi.org/10.1590/ES0101-73302015152420

Habermas, J. (1986). Problemas de Legitimación en el Capitalismo Tardío. Amorrortu.

Ley 18.962 de 1990. Ley Orgánica Constitucional de Educación. 10 de marzo de 1990.

Ley 20.248 de 2008. Ley de Subvención Escolar Preferencial. 1 de febrero de 2008.

Ley 20.370 de 2009. Ley General de Educación. 12 de septiembre de 2009.

Ley 20.529 de 2011. Sistema Nacional de Aseguramiento de la calidad de la educación parvularia, básica y media y su fiscalización. 27 de agosto de 2011

Ley 21.040 de 2017. Crea un nuevo Sistema de Educación Pública. 24 de noviembre de 2017. 
Muñoz, G. y Weinstein, J. (2019) The Difficult Process in Chile: Redefining the Rules of The Game for Subsidized Private Education. En C. Ornelas (Ed.), Politics of Education in Latin America (Vol. 49, pp.72-100). Brill Sense

Nueva Mayoría. (2013). Partidos, movimientos y coaliciones: Nueva Mayoría. https://www.bcn.cl/historiapolitica/partidos_politicos/wiki/Nueva_ Mayor\%C3\%ADa

Organización para la Cooperación y el Desarrollo Económicos. (2004). Revisión de Políticas Nacionales de Educación Chile. https://doi.org/10.1787/9789264021020-es

Offe, C. (1984). Contradictions in Welfare State. Hutchinson \& Co.

Orellana, V. (2018). Entre el Mercado Gratuito y la Educación Pública: Dilemas de la Educación Chilena Actual. LOM, Fundación Nodo XXI.

Rojas, M. (2014). Fin a la selección escolar: desafíos de una decisión necesaria. Cuaderno de Educación, (59), 1-7. https://repositorio.uahurtado.cl/ handle/11242/5680

Taylor, S., Rizvi, F., Lingard, B. y Henry, M. (1997). Educational Policy and the Politics of Change. Routledge.

Zancajo, A. (2019). Drivers and Hurdles to the Regulation of Education Markets: The Political Economy of Chilean Reform, Working Paper 239. http://ncspe.tc.columbia.edu/working-papers/WP239.pdf

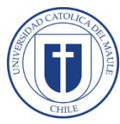

45

\section{Agradecimientos}

La elaboración de este artículo contó con financiamiento de la Agencia Nacional de Investigación y Desarrollo (ANID) / Subdirección de Capital Humano / Beca de Doctorado en el Extranjero Becas Chile 2020- 72210187.

\section{Dirección de correspondencia:}

Juan Antonio Carrasco-Bahamonde

Contacto: juanantonio.carrasco@e-campus.uab.ca 